\title{
3 Researchspuare \\ Fatal Nodular Regenerating Hyperplasia Rupture in a Common-Variable-Immunodeficiency Patient With TACI Mutation
}

Mongkhon Sompornrattanaphan

Mahidol University Faculty of Medicine Siriraj Hospital

Ranista Tongdee

Mahidol University Faculty of Medicine Siriraj Hospital

Chamard Wongsa

Mahidol University Faculty of Medicine Siriraj Hospital

Anupop Jitmuang

Mahidol University Faculty of Medicine Siriraj Hospital

Torpong Thongngarm ( $\nabla$ torallergy@gmail.com )

Mahidol University Faculty of Medicine Siriraj Hospital https://orcid.org/0000-0002-3182-4614

\section{Research Article}

Keywords: common variable immunodeficiency, hepatic rupture, immunodeficiency, nodular regenerating hyperplasia, TACI mutation

Posted Date: May 6th, 2021

DOl: https://doi.org/10.21203/rs.3.rs-470039/v1

License: (9) (1) This work is licensed under a Creative Commons Attribution 4.0 International License. Read Full License 


\section{Abstract}

\section{Background}

Nodular regenerating hyperplasia (NRH) is considered the most common liver involvement in common variable immunodeficiency (CVID). Most patients are asymptomatic with gradually increasing alkaline phosphatase (ALP) and mildly elevated transaminase enzymes over years. We report the first case of fatal NRH rupture complication in a CVID patient.

Case presentation

A 24-year-old man was diagnosed CVID at the age of 1.25 years. Genetic testing revealed a transmembrane activator and calcium-modulator and cyclophilin-ligand interactor (TACl) mutation. $\mathrm{He}$ had been receiving intravenous immunoglobulin (IVIg) replacement therapy ever since then. The trough level of serum IgG ranged between 750-1200 mg/dL. However, he still had occasional episodes of lower respiratory tract infection until bronchiectasis developed. At 22 years old, computed tomograph (CT) chest and abdomen as an investigation for lung infection revealed incidental findings of numerous nodular arterial-enhancing lesions in the liver and mild splenomegaly suggestive of $\mathrm{NRH}$ with portal hypertension. Seven months later, he developed sudden hypotension and tense bloody ascites. Emergency $\mathrm{CT}$ angiography of the abdomen showed NRH with intrahepatic hemorrhage and hemoperitoneum. Despite successful gel foam embolization, the patient died from prolonged shock and multiple organ failure.

\section{Conclusions}

Although CVID patients with NRH are generally asymptomatic, late complication including portal hypertension, hepatic failure, and hepatic rupture could occur. Therefore, an evaluation of liver function should be included in the regular follow-up of CVID patients.

\section{Background}

Common variable immunodeficiency (CVID) is characterized by impaired B-cell differentiation, causing decreased plasma cells and low levels of immunoglobulin production while circulating absolute $B$ cell counts and T cell functions are usually normal [1]. Clinical features of CVID are highly heterogeneous including infections primarily caused by bacteria in the respiratory and gastrointestinal tracts, autoimmune disorders despite the decreased antibody production, and malignancies. Immunoglobulin replacement therapy protects most patients against infection but does not protect them against noninfectious complications in which their prevalence are increasing as CVID patients live longer [2].

The prevalence of noninfectious liver diseases in a large CVID cohort in the United States (US) was 12.7\%, and granulomas and nodular regenerative hyperplasia $(\mathrm{NRH})$ were the most common pathological features [3]. The prevalence of abnormal liver function with raised alkaline phosphatase (ALP) levels in a 
CVID cohort in the United Kingdom (UK) was 43.5\%, mostly due to NRH [4]. Of interest, liver disease in CVID patients was associated with increased mortality [3]. NRH is common in CVID patients, yet fatal hepatic rupture due to NRH has never been reported. Herein, we report a 24-year-old male CVID patient with $\mathrm{NRH}$ who died due to hepatic rupture.

\section{Case Presentation}

A 24-year-old Thai male initially presented with recurrent sinopulmonary tract infection and chronic diarrhea since the age of 11 months old. Investigation of his immune status revealed an IgG level of 330 $\mathrm{mg} / \mathrm{dL}$ (normal range, $344-1180 \mathrm{mg} / \mathrm{dL}$ ) while absolute B cell (CD19) count was $1883 \mathrm{cells} / \mathrm{mm}^{3}$ (normal range, 171-465 cells $/ \mathrm{mm}^{3}$ ). His IgM and IgA were undetectable. The absolute CD4 and CD8 counts were 3050 cells $/ \mathrm{mm}^{3}$ (normal range, $1000-4600 \mathrm{~mm}^{3}$ ) and $2773 \mathrm{cells} / \mathrm{mm}^{3}$ (normal range, $400-2100 \mathrm{~mm}^{3}$ ), respectively. Genetic testing revealed a transmembrane activator and calcium-modulator and cyclophilinligand interactor (TACI) mutation.

The diagnosis of CVID was established at the age of 1.25 years. He had been receiving intravenous immunoglobulin (IVIg) replacement therapy ever since then. The trough level of serum IgG ranged between $750-1200 \mathrm{mg} / \mathrm{dL}$. However, he had occasional episodes of lower respiratory tract infection until he developed bronchiectasis at 15 years old.

At 22 years old, he developed an intermittent and remittent fever for 1 month. No causative organism was identified. Diagnostic workup including paranasal sinus films and nasal endoscopy were negative for sinusitis. Computed tomograph (CT) chest and abdomen suggested an active infectious process. Tuberculosis is common in Thailand, so empirical treatment with anti-tuberculous drugs was prescribed. Two months later, a repeat CT chest suggested improved pulmonary pathology. However, incidental findings of numerous nodular arterial-enhancing lesions in the liver were demonstrated. Multiphasic CT liver imaging and mild splenomegaly suggested NRH with early portal hypertension (Figure 1A-D). Seven months later, he was hospitalized with pneumonia. His IgG level was $890 \mathrm{mg} / \mathrm{dL}$. On the day 16 of the admission, he developed sudden hypotension. Physical examination revealed marked anemia and tense abdominal distension. Ultrasound-guided abdominal paracentesis yielded bloody fluid. Emergency CT angiography of the abdomen showed $\mathrm{NRH}$ with internal hemorrhaging and intramural pseudoaneurysm

(Figure 1E-F). Newly detected multiple areas of intrahepatic hemorrhage with rupture of the overlying Glisson's capsule, caused a large amount of hemoperitoneum. Although emergency angiography with gel foam embolization at the right and left hepatic artery was successful, the patient died from hemorrhagic shock and multiple organ failure.

The trends of aspartate transaminase, alanine transaminase, ALP, and serum IgG are summarized in Figure 2.

\section{Discussion And Conclusions}


We report a CVID patient experiencing a fatal hepatic rupture. Given the long period of ALP elevation and the $\mathrm{CT}$ imaging findings, $\mathrm{NRH}$ was the most likely diagnosis. Rupture is an uncommon complication of $\mathrm{NRH}$. To our knowledge, this is the first case to be reported as a complication of NRH in CVID patients.

Liver impairment was present in $11.9 \%$ of CVID patients in a US cohort [5]. Seventy-nine percent of CVID patients in a UK cohort had abnormal laboratories, imaging, and/or histopathology of liver disease [6]. $\mathrm{NRH}$ is generally considered the most common liver involvement in CVID with a prevalence of $5-12 \%$ [4, 7]. It is thought to be caused by intra-hepatic vasculopathy, leading to hepatocyte injury and regeneration of characteristic nodules [8]. Abundant regenerative nodules can compress hepatic sinusoids, causing noncirrhotic portal hypertension [7]. Most patients are asymptomatic with gradually increasing ALP levels over years [4]. Mildly elevated transaminases were reported in approximately $50 \%$ of patients [7]. Late complications include ascites, esophageal varices, and splenomegaly due to portal hypertension, usually appearing years after the elevated liver enzyme [4, 7]. CT features of NRH are multiple, arterial-enhancing liver nodules or masses typically becoming iso- or slightly hyperintense in the portovenous and delayed phases without rapid washout [9]. The most common histopathological finding in CVID patients with $\mathrm{NRH}$ is intrasinusoidal inflammatory cell infiltrates with CD3, CD4, and CD8 T cells which is suggestive of autoimmune process [7]. Chronic CD8 cell infiltration would partially account for increased IFNg production which positively correlated with disease severity [7]. NRH could be associated with autoimmune cytopenia, polyclonal lymphoproliferation, and diffuse granulomatous disease whereas no associations were found with age at onset, age at diagnosis, and duration of immunoglobulin replacement therapy [4].

Differential diagnosis for multiple hypervascular hepatic lesions includes malignant hypervascular tumors, e.g., hepatocellular carcinoma (HCC) and benign lesions, e.g., hepatic adenoma (HA), hemangioma, and $\mathrm{NRH}$. Although HCC is the most common hypervascular liver tumor causing spontaneous hepatic rupture, the absence of typical CT findings for HCC including early enhancement with rapid washout in portovenous or delayed phase makes the diagnosis unlikely in the present case. HA is commonly seen in young women and is associated with oral contraceptives or anabolic steroid use, making $\mathrm{HA}$ an unlikely diagnosis in the present case although the CT findings may support this diagnosis. Hemangioma typically shows peripheral nodular enhancement in the arterial phase and progressive contrast fill-in in the delayed phase. None of those findings were seen in the present case.

In conclusion, NRH is the most common liver involvement in CVID patients. Most CVID patients with NRH are asymptomatic with gradually increasing ALP levels. NRH could lead to portal hypertension and a fatal rupture complication as in the present case. Therefore, an evaluation of liver function should be included in the follow-up of CVID patients.

\section{Declarations}

\section{Ethics approval, consent to participate}

Written and informed consent was obtained from the patient's relative to participate. 


\section{Consent for publication}

Written and informed consent for publication was obtained from the patient's relative. The patient's relative was informed that de-identified data would be used in the scientific research and publications.

\section{Availability of data and materials}

Not applicable

\section{Competing interests}

The authors declare that they have no competing interests.

\section{Funding}

No funding has been received for this manuscript.

\section{Authors' contributions}

MS, RT, CW, AJ and TT each contributed to the laboratory analysis and interpretation of the data, and revised the manuscript for important scientific content. All authors read and approved the final manuscript.

\section{Acknowledgements}

We acknowledge Dr Anthony Tan for editing the English language in the manuscript.

\section{References}

1. Gathmann B, Mahlaoui N, Ceredih, Gerard L, Oksenhendler E, Warnatz K, et al. Clinical picture and treatment of 2212 patients with common variable immunodeficiency. J Allergy Clin Immunol. 2014;134(1):116-26.

2. Farmer JR, Ong MS, Barmettler S, Yonker LM, Fuleihan R, Sullivan KE, et al. Common Variable Immunodeficiency Non-Infectious Disease Endotypes Redefined Using Unbiased Network Clustering in Large Electronic Datasets. Front Immunol. 2017;8:1740.

3. Ho HE, Cunningham-Rundles C. Non-infectious Complications of Common Variable Immunodeficiency: Updated Clinical Spectrum, Sequelae, and Insights to Pathogenesis. Front Immunol. 2020;11:149.

4. Ward C, Lucas M, Piris J, Collier J, Chapel H. Abnormal liver function in common variable immunodeficiency disorders due to nodular regenerative hyperplasia. Clin Exp Immunol. 2008;153(3):331-7.

5. Cunningham-Rundles $C$, Bodian C. Common variable immunodeficiency: clinical and immunological features of 248 patients. Clin Immunol. 1999;92(1):34-48. 
6. Azzu V, Fonseca M, Duckworth A, Kennard L, Moini N, Qurashi M, et al. Liver disease is common in patients with common variable immunodeficiency and predicts mortality in the presence of cirrhosis or portal hypertension. J Allergy Clin Immunol Pract. 2019;7(7):2484-6 e3.

7. Fuss IJ, Friend J, Yang Z, He JP, Hooda L, Boyer J, et al. Nodular regenerative hyperplasia in common variable immunodeficiency. J Clin Immunol. 2013;33(4):748-58.

8. Morris JM, Oien KA, McMahon M, Forrest EH, Morris J, Stanley AJ, et al. Nodular regenerative hyperplasia of the liver: survival and associated features in a UK case series. Eur $\mathrm{J}$ Gastroenterol Hepatol. 2010;22(8):1001-5.

9. Brancatelli G, Federle MP, Grazioli L, Golfieri R, Lencioni R. Large regenerative nodules in Budd-Chiari syndrome and other vascular disorders of the liver: CT and MR imaging findings with clinicopathologic correlation. AJR Am J Roentgenol. 2002;178(4):877-83.

\section{Figures}




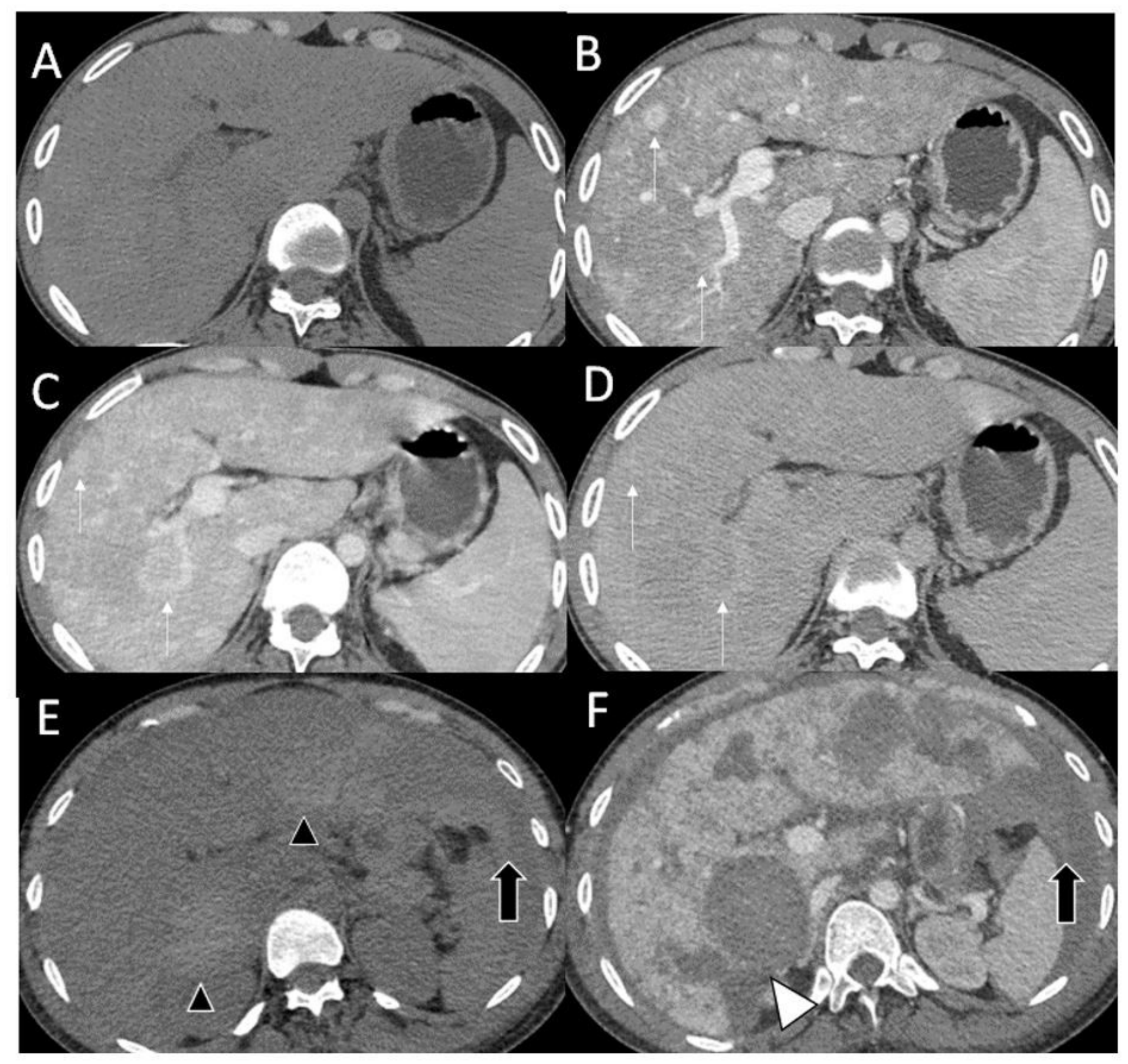

\section{Figure 1}

Hepatic imaging A-D. The initial multiphasic CT. A) The noncontrasted image shows no abnormal intrahepatic bleeding. B) The arterial phase shows multiple poorly-defined, arterial-enhancing nodules scattered throughout both hepatic lobes (white arrows). In portovenous phase $\mathrm{C}$ ) and 5 min-delayed phase $\mathrm{D}$ ), these nodules remain iso- to hyperatternuating compared to the adjacent liver parenchyma. Hepatic imaging E-F. The 7-month follow-up CT. E) The non-contrasted image shows multiple areas of intrahepatic bleeding (black arrow heads) and intraperitoneal bleeding (black arrows). F) The portovenous phase demonstrates disruption of the liver capsule (white arrow head), suggesting rupture of these hypervascular liver lesions. 


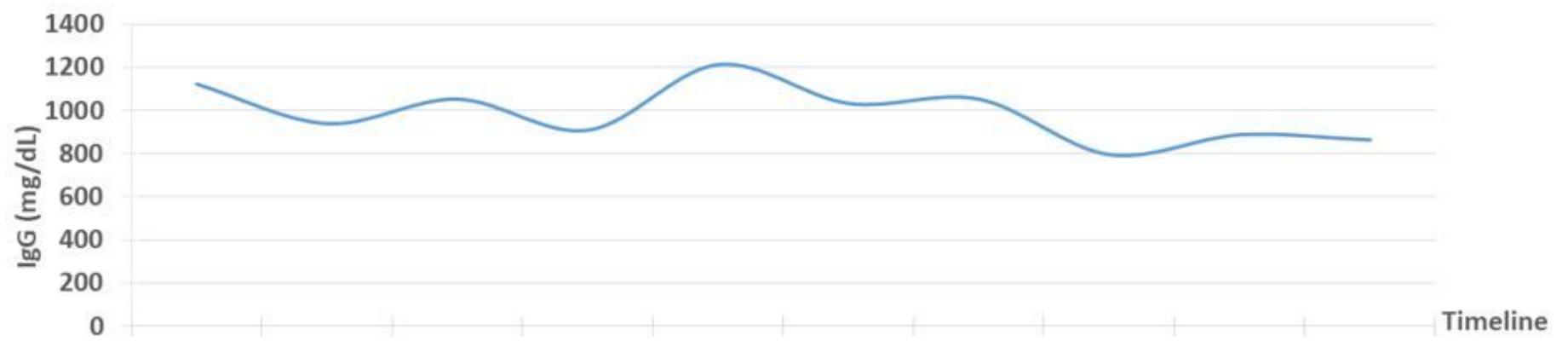

Jun 2015 Dec 2015 May 2016 Oct 2016 Jan 2017 Sep 2018 May 2019 Jul 2019 Sep 2019 Oct 2019

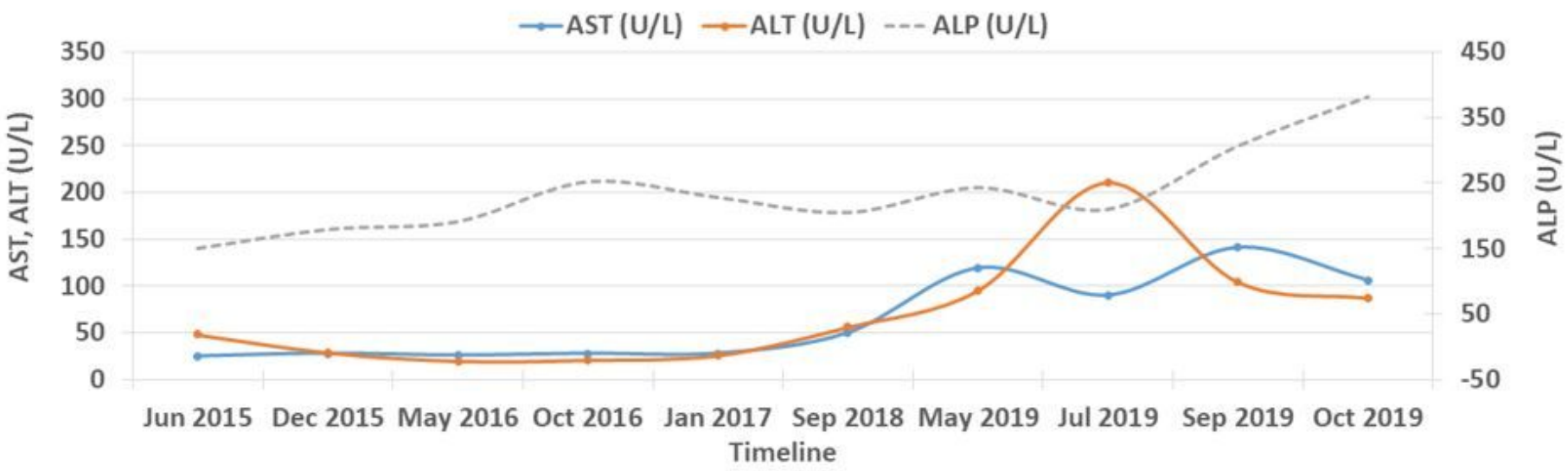

Figure 2

The trend of liver enzymes and trough level of immunoglobulin $\mathrm{G}$ during follow-up Abbreviation: ALP, alkaline phosphatase; AST, aspartate transaminase; ALT, alanine transaminase; IgG, immunoglobulin G 